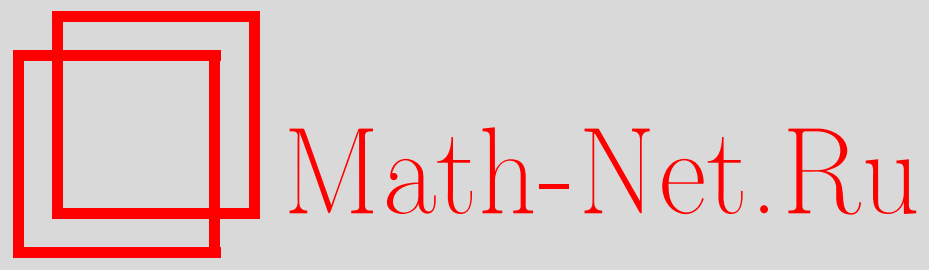

В. В. Петров, О законе повторного логарифма для последовательности независимых случайных величин, Теория вероятн. и ее примен., 2001, том 46, выпуск 3, 569-571

DOI: https://doi.org/10.4213/tvp3903

Использование Общероссийского математического портала Math-Net.Ru подразумевает, что вы прочитали и согласны с пользовательским соглашением http://www . mathnet.ru/rus/agreement

Параметры загрузки:

IP: 54.162 .127 .20

26 апреля 2023 г., 12:14:27

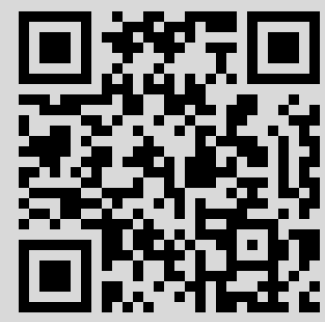


(C) $2001 \mathrm{r}$.

ПЕTPOB B. B.*

\title{
О ЗАКОНЕ ПОВТОРНОГО ЛОГАРИФМА ДЛЯ ПОСЛЕДОВАТЕЛЬНОСТИ НЕЗАВИСКМЫХ СЛУЧАЙНЫХ ВЕЛИЧИН ${ }^{1)}$
}

\begin{abstract}
Исследованы условия применимости закона повторного логарифма к последовательности независимых случайных величин с конечными дисперсиями.
\end{abstract}

Ключевые слова и фразы: закон повторного логарифма, последовательности независимых случайных величин.

1. Введение. Рассмотрим последовательность независимых случайных величин $X_{1}, X_{2}, \ldots$ с конечными дисперсиями и математическими ожиданиями, равными нулю. Положим

$$
\begin{gathered}
S_{n}=\sum_{j=1}^{n} X_{j}, \quad B_{n}=\sum_{j=1}^{n} \mathbf{E} X_{j}^{2}, \\
F_{n}(x)=\mathbf{P}\left\{B_{n}^{-1 / 2} S_{n}<x\right\}, \quad \Phi(x)=(2 \pi)^{-1 / 2} \int_{-\infty}^{x} e^{-t^{2} / 2} d t .
\end{gathered}
$$

В работе автора [1] (см. также [2, с. 261]) показано, что если выполнены условия ${ }^{2)}$

$$
\begin{aligned}
B_{n} & \rightarrow \infty, \\
\frac{B_{n+1}}{B_{n}} & \rightarrow 1, \\
\sup _{x}\left|F_{n}(x)-\Phi(x)\right| & =O\left(\left(\ln B_{n}\right)^{-1-\delta}\right)
\end{aligned}
$$

при некотором $\delta>0$, то

$$
\limsup \frac{S_{n}}{\left(2 B_{n} \ln \ln B_{n}\right)^{1 / 2}}=1 \text { п.н. }
$$

В этом предложении нельзя ослабить условие (3), заменив в нем $\delta>0$ значением $\delta=0$. В. А. Егоров [3] построил пример последовательности независимых случайных величин, удовлетворяющей условиям (1), (2) и условию (3) при $\delta=0$, для которой соотношение (4) не выполнено. Представляет интерес исследование эффекта нарушения условия (2).

2. Основной результат. Целью настоящей работы является доказательство следующей теоремы.

Теорема. Пусть $X_{1}, X_{2}, \ldots$ - последовательность независимых случайньх величин с математическими ожиданиями, равными нулю, и конечньми дисперсиями. Если выполнены условия (1) и (3) при некотором $\delta>0$, то

$$
\limsup \frac{S_{n}}{\left(2 B_{n} \ln \ln B_{n}\right)^{1 / 2}} \leqslant 1 \quad \text { п.н. }
$$

* С.-Петербургский государственный университет; математико-механический факультет, Старый Петергоф, 198904 С.-Петербург, Россия.

1) Работа выполнена при поддержке Российского фонда фундаментальных исследований (грант № 99-01-00732).

2) Пределы указаны всюду при $n \rightarrow \infty$, если не оговорено противное. 
3. Доказательство. Пусть $\tau-$ произвольное положительное число. Используя условие (1) и неубывание последовательности $\left\{B_{n}\right\}$, определим последовательность цельіх положительных чисел $\left\{n_{k} ; k=1,2, \ldots\right\}$ следующим образом: пусть $n_{1}$ таково, что $B_{n_{1}}>e$; при данном $n_{k-1}(k=2,3, \ldots)$ определим $n_{k}$ как наименьшее целое число, для которого $B_{n_{k}}>(1+\tau) B_{n_{k-1}}$. Таким образом, справедливы неравенства

$$
B_{n_{k}-1} \leqslant(1+\tau) B_{n_{k-1}}<B_{n_{k}} .
$$

Из (6) следует, что $n_{k-1}<n_{k}$ при всех $k \geqslant 2$, так что последовательность $\left\{n_{k}\right\}$ строго возрастает и $n_{k} \rightarrow \infty$ при $k \rightarrow \infty$.

Положим $L(n)=\left(2 B_{n} \ln \ln B_{n}\right)^{1 / 2}, \bar{S}_{n}=\max _{1 \leqslant j \leqslant n} S_{j}$. Соотношение (5) будет доказано, если мы покажем, что

$$
\mathbf{P}\left\{S_{n}>(1+\varepsilon) L(n) \text { б.ч. }\right\}=0
$$

для любого $\varepsilon>0$. Докажем сначала, что

$$
\sum_{k} \mathbf{P}\left\{\bar{S}_{n_{k}-1}>(1+\varepsilon) L\left(n_{k-1}\right)\right\}<\infty
$$

для любого $\varepsilon>0$. С этой целью применим неравенство Колмогорова $\mathbf{P}\left\{\bar{S}_{n} \geqslant x\right\} \leqslant$ $2 \mathbf{P}\left\{S_{n} \geqslant x-\left(2 B_{n}\right)^{1 / 2}\right\}$, справедливое для сумм независимых случайных величин с конечными дисперсиями и математическими ожиданиями, равными нулю, и для любого $x$ (см., например, [2, с. 79]). Для всех достаточно больших $k$ имеем

$$
\begin{aligned}
\mathbf{P}\left\{\bar{S}_{n_{k}-1}>(1+\varepsilon) L\left(n_{k-1}\right)\right\} & \leqslant 2 \mathbf{P}\left\{\bar{S}_{n_{k}-1}>(1+\varepsilon) L\left(n_{k-1}\right)-\left(2 B_{n_{k}-1}\right)^{1 / 2}\right\} \\
& \leqslant 2 \mathbf{P}\left\{S_{n_{k}-1}>(1+\gamma) L\left(n_{k}-1\right)\right\}
\end{aligned}
$$

для любого $\gamma<\varepsilon$, так как

$$
\begin{aligned}
(1+\varepsilon) L\left(n_{k-1}\right)-\left(2 B_{n_{k}-1}\right)^{1 / 2} & \geqslant \frac{1+\varepsilon}{(1+2 \tau)^{1 / 2}} L\left(n_{k}-1\right)-\left(2 B_{n_{k}-1}\right)^{1 / 2} \\
& \geqslant(1+\gamma) L\left(n_{k}-1\right)
\end{aligned}
$$

в силу (6) для любого $\gamma<\varepsilon$, если взять $\tau$ достаточно малым.

Используя соотношение $1-\Phi(x)=(2 \pi)^{-1 / 2} x^{-1} e^{-x^{2} / 2}(1+o(1))$ при $x \rightarrow+\infty$, получаем

$$
1-\Phi\left(b\left(2 \ln \ln B_{n}\right)^{1 / 2}\right)=(2 b)^{-1}\left(\pi \ln \ln B_{n}\right)^{-1 / 2}\left(\ln B_{n}\right)^{-b^{2}}(1+o(1))
$$

для любого $b>0$. Отсюда и из равенства $\mathbb{P}\left\{S_{n} \geqslant b L(n)\right\}=1-\Phi\left(b\left(2 \ln \ln B_{n}\right)^{1 / 2}\right)+$ $O\left(\left(\ln B_{n}\right)^{-1-\delta}\right)$, вытекаюего из условия (3), находим, что

$$
\mathbf{P}\left\{S_{n} \geqslant b L(n)\right\} \leqslant\left(\ln B_{n}\right)^{-b^{2}}
$$

для любого положительного $b<(1+\delta)^{1 / 2}$ и всех достаточно больших $n$.

В силу (6) имеем

$B_{n_{k}-1} \geqslant B_{n_{k-1}} \geqslant(1+\tau)^{k-2} B_{n_{1}} \geqslant(1+\tau)^{k-2} e, \quad\left(\ln B_{n_{k}-1}\right)^{-b^{2}} \leqslant((k-2) \ln (1+\tau))^{-b^{2}}$

для любого целого $k \geqslant 3$. Из (9) и (10) следует, что

$$
\mathbf{P}\left\{\bar{S}_{n_{k}-1}>(1+\varepsilon) L\left(n_{k-1}\right)\right\} \leqslant M(k-2)^{-c^{2}}
$$

для всех достаточно больших $k$, если $c^{2}<\min \left\{(1+\varepsilon)^{2}, 1+\delta\right\}$. Здесь $M-$ положительная постоянная, не зависящая от $k$. При этом для данных положительных $\varepsilon$ и $\delta$ постоянную $c$ можно выбрать удовлетворяющей условию $c>1$. Поэтому из (11) следует (8). 
Для люоого $\varepsilon>0$ имеем

$$
\begin{aligned}
\mathbf{P}\left\{S_{n}>(1+\varepsilon) L(n) \text { б.ч. }\right\} & \leqslant \mathbf{P}\left\{\max _{n_{k-1 \leqslant n<n_{k}}} S_{n}>(1+\varepsilon) L\left(n_{k-1}\right) \text { б.ч. }\right\} \\
& \leqslant \mathbf{P}\left\{\bar{S}_{n_{k}-1}>(1+\varepsilon) L\left(n_{k-1}\right) \text { б.ч. }\right\}=0
\end{aligned}
$$

в силу (8) и леммы Бореля-Кантелли. Равенство (7) доказано. Теорема доказана.

4. Замечание. Если последовательность независимых случайных величин удовлетворяет условиям (1) и (3) при некотором $\delta>0$, но не удовлетворяет условию (2), то соотношение (4) может не выполняться.

Рассмотрим последовательность независимых нормально распределенных случайных величин $\left\{X_{n}\right\}$ такую, что $\mathbf{E} X_{n}=0$ и $B_{n}=\exp \left\{n^{\beta}\right\}$, где $\beta>1$. Для этой последовательности выполнены условия (1) и (3) при любом $\delta>0$, так как $F_{n}(x) \equiv \Phi(x)$ при любом $n$. Условие (2) не выполнено. Используя рассуждения, аналогичные приведенным в п. 3 , при $n_{k}=k$, нетрудно показать, что $\lim \sup S_{n}\left(2 B_{n} \ln \ln B_{n}\right)^{-1 / 2} \leqslant$ $\beta^{-1 / 2}$ п.н. (Можно также доказать непосредственно или с помощью одного результата Коно [4], что здесь имеет место знак равенства.) Соотношение (4) не выполнено, поскольку $\beta>1$.

\title{
СІИСОК ЛИТЕРАТУРЫ
}

1. Петров В. В. О связи между оценкой остаточного члена в центральной предельной теореме и законом повторного логарифма. - Теория вероятн. и ее примен., 1966 , т. 11 , в. 3 , с. $514-518$.

2. Петров В. В. Предельные теоремы для сумм независимых случайных величин. М.: Наука, 1987, 317 с.

3. Егоров B. A. О законе повторного логарифма. - Теория вероятн. и ее примен., 1969 , т. 14 , в. 4 , c. $722-729$.

4. Kôno $N$. Une extension de la loi du logarithme itéré pour la somme de variables aléatoires indépendantes et gaussiennes centrées. - C. R. Acad. Sci. Paris, 1974, v. 279 , № 9, p. 345-346.

(C) $2001 \Gamma$.

СОФРОНОВ Г. Ю.*

\section{АСИМПТОТИЧЕСКИ $d$-ОПТИМАЛЬНЫЙ КРИТЕРИЙ ОБНАРУЖЕНИЯ РАЗЛАДКИ}

\begin{abstract}
Рассматривается задача обнаружения разладки для последовательности случайных величин. Строится $d$-оптимальный критерий, гарантирующий $d$-риск. Найдена асимптотика этого критерия.

Ключевые слова и фразы: обнаружение разладки, $d$-апостериорный подход, $d$-гарантийность, $d$-оптимальность, слабая сходимость, функционалы от винеровского процесса.
\end{abstract}

Пусть $X^{(n)}=\left(X_{1}, X_{2}, \ldots, X_{n}\right)$ - случайный вектор с независимыми компонентами. Рассматривается задача проверки гипотезы $H_{0}$ (отсутствие разладки): независимые случайные величины $X_{1}, \ldots, X_{n}$ ( $n$ фиксировано) одинаково распределены с плотностью $f(x)$, при альтернативе $H_{1}$ (есть разладка): $X_{1}, \ldots, X_{\tau}$ распределены

* Марийский государственный университет, Кафедра матем. анализа и теории функций, Йошкар-Ола. 